\title{
Determinates of ANC in Deciding Place of Delivery for Marginalized Women in Rural Odisha, India-Women's Perspective
}

\author{
${ }^{1}$ Meerambika Mahapatro, ${ }^{2}$ Shalini Kelkar \\ ${ }^{1}$ Associate Professor, National Institute of Health and Family Welfare, New Delhi \\ ${ }^{2}$ Senior Medical Officer, Directorate of Health Services, GNCT, Delhi \\ Email: Meerambika.mahapatro@gmail.com,meerambika@rediffmail.com
}

\begin{abstract}
Antenatal care (ANC) is very important intervention needed for management of normal cases of pregnancy as well as for screening and detection of complications at an earlier stage so as to manage them effectively in time. This article has tried to highlight the factors that are proved to be the driving and restraining forces in accessing antenatal care services in India and how it influences the women to take decision for the place of delivery (home or institutional). A mixed method approach was applied to 320 women from 16 villages of Ganjam and Gajapati districts of Odisha. Indepth interviews were carried out till data saturation was observed at 10 with women having child of less than one year. 10 Focus Group Discussion were conducted with an average of 8 women in each group having a child of less than 5 years of age, and with the community. In-depth interview of women regarding their antenatal checkups revealed that $55.63 \%$ had visited the ANC clinics 3 or more times, $42 \%$ were primi and $58 \%$ were multipara. A large mass of population still had not taken full antenatal checkup in-spite of all the available health facilities at their door step. The infrastructure of the government health facilities needs improvement. Access to health services has to be guaranteed to all people irrespective of rural or urban divide. Where patriarchal traditional values still prevail, it is often difficult for women, to use health services unless the provided services are culturally acceptable in practice. The most important and challenging need of the hour is 'women empowerment' in the society to access their rights.
\end{abstract}

Key words: Antenatal care, accessibility, utilization, autonomy, decision-making.

\section{Introduction}

Antenatal care is systematic supervision of women during pregnancy to monitor the progress of foetal growth and to ascertain the well-being of the mother and the foetus. Attendance of four antenatal care (ANC) checkups include identification and management of obstetric complications, routine tetanus toxoid immunization, iron and folate supplementation and identification and management of infections resulting in reduction of maternal deaths and improvement in reproductive health $[1,2,3]$. The pregnant women need care during this critical period for their well-being and for the health of the babies they are bearing. ANC comprises - careful history and current state examinations (general and obstetrical), management of major and minor problems and advice being given to the pregnant women. It is the single most important intervention which can reduce the maternal and infant (especially neonatal) -morbidity and mortality. ANC is an opportunity to promote the use of skilled attendance at birth and to decide the place of delivery i.e. institutional or home delivery. It also promotes healthy behaviours such as breastfeeding, early postnatal care, and planning for optimal pregnancy spacing $\lceil 4,5]$.

The antenatal care facilities provided by the Indian government can be accessed by visiting a health centre that provides such facilities. National Family Health Survey -3 (2005-2006) report [6] showed that only $50.7 \%$ of women had at least 3 antenatal care visits for their last birth, $73.8 \%$ in urban areas and $42.8 \%$ in rural areas. Also this figure varied from $29.8 \%$ with no education to $85.3 \%$ with 10 or more years of education. Literature shows that men's involvement in antenatal care in NFHS-3, where men were questioned about the main reason for not receiving antenatal care by the child's mother, $40.4 \%$ of men said that according to them it was not necessary or they did not allow their wives. There was not much difference in this figure between urban and rural areas as $38.8 \%$ of men in urban areas and $40.7 \%$ 
in rural areas gave this reason. With government antenatal care services in place along with various schemes like Janani Suraksha Yoiana(JSY) 「7〕 and Indira Gandhi Matritva Sahyog Yoiana (IGMSY) [8], it is important to look at the reasons for under/ non-utilization of these services. JSY is a safe motherhood intervention being implemented with the objective of reducing maternal and neo-natal mortality by promoting institutional delivery among the poor pregnant women. While IGMSY is a conditional cash transfer scheme for pregnant and lactating women to provide conditions for safe delivery and good nutrition and feeding practices. To fully benefit from these interventions, it is important that women begin attending ANC early in pregnancy. Many of these opportunities continue to be missed, even though over two-thirds of pregnant women receive at least one antenatal visit. There are many reasons or barriers to reach to the health care center for the women [9]. To strengthen ANC as priority intervention to increase coverage and quality, the Indian government introduced the Accredited Social Health Activist (ASHA) programme under the National Rural Health Mission (NRHM) [10].

A study done to understand the user's perspectives on barriers to maternal health care use in Maharashtra [11], India found that socioeconomic status was not a barrier to service use when women perceived the benefits of the service, and when the service was available near the user's place of residence. Most women considered private health services to be superior to those provided by the government, although cost was often a constraint to their use. Another finding from the study was that antenatal care and institutional delivery were both considered to be preventative measures rather than curative, hence were not prioritized due to financial constraints. A meta-analysis of qualitative studies done in various low and middle-income countries including India identified three main themes based on views and experiences of women who had received inadequate antenatal care in these countries [5]. According to the theme, many women had cultural and superstitious beliefs that if they visit an antenatal clinic, their pregnancy would be disclosed which might lead to unwanted religious or spiritual complications, curses of evil spirits, indirect cost to visit the health centre, no quality health care and long waiting time [12]. Another common reason given for not accessing antenatal care services was the bad attitude of staff at health care facilities that included insensitivity, rude behaviour, humiliation, neglect, abuse and even physical violence by health care providers. Some of the women visited antenatal clinic only once to get the 'card' made so that they would not be denied when services are needed especially at the time of delivery.

Odisha is one of the eight Empowered Action Group (EAG) states recognized by the Government of India (GOI) for special care in terms of Health and Family Welfare services [10]. Being an EAG state, it receives added focus and incentive from the Government. Basic training is being given to health workers at all levels of health care so that they can make important decisions regarding how to treat, when to treat, where to treat, what to treat and whom to treat, under various MCH programs. The 20th century was marked by a significant improvement in access to healthcare, worldwide. However, the delivery of these services to the beneficiaries was not completely or uniformly distributed among, or even within, nations and was faced with a considerable amount of road blocks leading to heightened MMR (237 per 1 lakh live birth) and IMR (62 per 1000 live births). Despite the fact that the state government has taken many initiatives to converge health activities at village and subsequent levels, this problem is still so difficult to address in India that making safe motherhood a reality for women is a challenge that calls for re-conceptualization of the problem and its solution. This article has tried to highlight the factors that prove to be the driving and restraining forces in accessing the antenatal care services in India and how it influences women to take decisions regarding the place of delivery (home or institutional).

\section{Material and Methods}

\subsection{Study Design}

The study was carried out using mixed method; both Quantitative \& Qualitative methods were used. Descriptive cross-sectional method was used as quantitative study design.

\subsection{Population (Inclusion Criteria)}

- Women delivered in last one year and the pregnant women 
- Women delivered in last 5 years

- $\quad$ Service Providers (ASHA, AWW, ANM, LHV, Staff Nurse, MO, CDMO, CDPO)

- Key Influencers, Decision Makers (Sarpanch, Mahila Mandal, Voluntary Health and Sanitation Committee members, BDO, Tahsildar).

\subsection{Area}

Ganjam and Gajapati districts of Orissa were selected for the study. A total of 16 villages from 2 districts were considered.

\subsection{Sample Design and Sample Size}

A convenience purposive sampling was adopted. One Ganjam district which has the medical college and hospital and the other district Gajapati which was nearer to a medical college and hospital were selected. Two blocks, one performing high and the other performing low on the indicators of antenatal care/antenatal registration by 12 weeks, skilled birth attendant, institutional delivery, utilization of JSY and postnatal visit, as per RCH-II data, were selected from each district. Within that, two sub-centers villages were selected from each block; one village with sub-centre and the other located at least $5 \mathrm{~km}$ from the sub-centre (out-reach village). There are about 150-180 eligible couples per 1000 population or the under 5 children composition is 11 to $13 \%$ of population. Considering that there should be 110-130 mothers in the population, we have taken $10 \%$ of that i.e. 11-13 mothers. Therefore, a total of 20 mothers from each village were considered. A total of 320 women from 16 villages were considered for the study.

\subsection{Data Collection Tools and Techniques}

The sources of information included both primary and secondary data. Primary data for the study was collected by way of detailed interview of respondents through the semi structured interview schedules specifically designed. In-depth Interview was focused on perspective and practices of women having oneyear infant(s) or having delivered in the last one year regarding evidence based practices, postnatal and newborn care in order to ensure that responses reflected recent/current practices. The concerned health care providers also constituted the main source of information regarding the implementation of services in the study area. These interview schedules were finalized after several rounds of discussion \& pretesting and then applied to the study population to know their perception regarding the utilization of MCH services. The information from the stakeholders CMOs, MOs, ASHA, ANM, health worker, PRI members, V HSC, SHGs, community leaders, other health professionals etc. $\}$ was collected through detailed interviews. Key Informant Interviews (KII) were conducted with the providers and health workers, traditional birth attendants (TBAs), ASHA, and ANM of health services to present their own experiences and actions, or otherwise to describe general attitude. Focus Group Discussions (FGD) were conducted focusing on the utilization of ANC practices, birth preparedness, skilled attendants at delivery, postpartum care for the mother and baby, acceptability of home visits by community volunteers, etc. among women who delivered in the last five years and the grass-root level health workers. FGDs and Interviews with KIs were conducted in local language. Secondary data from the districts and selected health facilities were used. Available reports and records regarding the following were reviewed.

\subsection{Data Analysis}

Analysis of the quantitative data was done using a software package to calculate Proportion \& percentage, Pearson Chi-square, etc. The data hereforth collected was analyzed using SPSS software program in the Dept. of SPM, MKCG Medical College Berhampur which came out with valuable recommendations. Latent thematic content analysis was done on the qualitative data: like in-depth interviews (IDIs) and FGDs. The data collected in the form of recorded interviews was coded and each interview was transcribed. The data was also triangulated with the help of field notes. Transcripts of the interviews with mothers, health workers, and key informants were reviewed together. The data was 
reviewed and transcripts were reread to identify important themes. Then the patterns, possible relationships between themes, contradictory responses were identified. The statements in the interviews were categorized and analyzed according to frequency in general, frequency in each different group of interview, controversial discussion of the statement and emotional involvement of the interview participants. Typical statements were marked and used for later citation. Analysis, interpretation and conclusions were carefully drawn describing the research context and the characteristics of the study participants.

\subsection{Ethical Aspect}

The written consent of the participants was taken and the study was ethically approved from Institutional Review Board, NIHFW.

\section{$3 \quad$ Result and Discussion}

We have analyzed the information elucidated from 320 women regarding the determinants to using health care facility for antenatal care and how it influenced the women to take decisions for the place of delivery (home or institutional). Form qualitative data, force field analysis was done which depicts the driving forces and restraining forces for accessibility and utilization of ANC care. The determinants of accessing ANC care were analyzed using 5 indicators such as education, income, parity, involvement of men and equity (table 1). In depth interview of women regarding their antenatal checkups revealed that $55.63 \%$ had taken 3 or more ANC visits. A large mass of population had still not taken full antenatal checkup inspite of all the available health care facilities at their door step.

\subsection{Education and ANC}

The rate of $>3 \mathrm{ANC}$ visits among literate women was higher (83\%) as compared to illiterate women. This infers that the literacy status is directly proportional to number of antenatal visits made by the women. [Chi square $=140.87$ with 1 degree of freedom and the two-tailed $p$ value $<0.0001]$. The association between literacy status and $>3$ antenatal checkups was considered to be extremely statistically significant.

\subsection{Income and ANC}

It was observed that income does not influence the number of ANC visits (table 1). Among women belonging to $<$ Rs. 3000 income group, approximately 77 percent had made more than three ANC visits. It showed that the influence of grass root level workers and increased awareness among the women led to better antenatal care services utilization.

\subsection{Parity and ANC}

Among the study population, $42 \%$ were primipara and $58 \%$ were multipara. The rate of $>3$ ANC visits were higher among primiparous women whereas it was only $62 \%$ among multiparous women. This indicates that parity was inversely proportional to the rate of antenatal visits made by women. $[C h i$ square $=52.843$, with one degree of freedom. The two-tailed $p$ value was less than 0.0001]. The association between parity and $>3 \mathrm{ANC}$ visits was considered to be extremely statistically significant.

\subsection{Involvement of Men}

Women were asked regarding their husband's involvement in receiving antenatal care. The reason given by the women was that the family did not think ANC was necessary, it was a women issue, therefore, men should not go along with her to the health centre. ANC is a novel phenomenon to women and they have had children before also, so why receive ANC now, with additional out-of-pocket expenses. Key Influencers of Phulta were of the opinion that the pregnant women can't even come to anaganwadi centre for collection of supplementary diet as they will not be allowed by the elder women of the family. 
The reason is that someone may do black magic. It was also reported that women had to ask for permission from the husband or head of the household to leave their home, including making a visit to health facilities.

\subsection{Equity in Accessing ANC due to Locational Advantage (Remote Village and Sub- Centre Village)}

A remote village in India doesn't have a sub-centre and is $5 \mathrm{kms}$ away from the sub-centre village, while a sub-centre village has one sub-centre where basic maternal health services are provided. The subcentre village is predominated by the tribals whereas the remote village is dominated by the schedule castes, the marginalized community. The demographic indicators were compared with both villages. It was found that women receiving $<3 \mathrm{ANC}$ are more (14\%) distributed in the remote village and women receiving $>3 \mathrm{ANC}$ are more, about 44\%, distributed in the sub-centre village. It is found to be significant. It can be concluded that irrespective of their caste, it is the location of the health center which brings the differentiation in accessing ANC services.

Table 1: Variables used in modeling ANC care

\begin{tabular}{|c|c|c|c|}
\hline Variables & $<3 \mathrm{ANC}$ & $>3 \mathrm{ANC}$ & Chi square $\&$ p $p$ value \\
\hline \multicolumn{3}{|l|}{ Education } & \multirow[t]{3}{*}{ Chi square $=140.87 p$ value $<0.001$} \\
\hline Illiterate & (134) $83.7 \%$ & (26) $16.2 \%$ & \\
\hline literate & (27) $16.9 \%$ & (133) $83.1 \%$ & \\
\hline \multicolumn{3}{|l|}{ Income (in Rs.) } & \multirow[t]{4}{*}{ Not significant } \\
\hline$<3000$ & (42) $64.6 \%$ & (195) $76.8 \%$ & \\
\hline $3001-5000$ & (21) $30.8 \%$ & (41) $16.1 \%$ & \\
\hline$>5001$ & (3) $4.6 \%$ & (18) $7.1 \%$ & \\
\hline \multicolumn{3}{|l|}{ Parity } & \multirow[t]{3}{*}{ Chi square $=52.843, p$ value $<0.001$} \\
\hline Primiparous & (34) $21.3 \%$ & (126) $78.7 \%$ & \\
\hline Multiparous & (99) $61.9 \%$ & (61) $38.1 \%$ & \\
\hline \multicolumn{3}{|l|}{ Location of health centre } & \multirow{3}{*}{$\begin{array}{l}\text { Chi square }=11.880 \\
p \text { value }<0.001\end{array}$} \\
\hline Sub-centre village & (45) $28.1 \%$ & (21) $12.6 \%$ & \\
\hline Remote village & $71.9 \%$ & (139) $87.4 \%$ & \\
\hline
\end{tabular}

Data from the qualitative analysis presented in the figure depicts the Force Field Analysis of Utilization of Antenatal Care Services by women which influences their choice between institutional or home delivery. The driving forces for accessing and utilizing ANC by the pregnant women which influence them for deciding institutional delivery are free ANC services, JSY and IGMSY cash benefit schemes for beneficiaries provided by the government, and the persuasion from grass-root health workers. While low education, low income, unavailability of transport system, loss of wages, additional indirect cost, partly or non-involvement of men folks of the family, superstitious beliefs, etc are the restraining forces. Comparison of the two forces shows that restraining forces are dominant over driving forces which, therefore, impede decision of the women for institutional delivery.

The village is far away from the hospital and main road. At night transportation is more difficult as we have to cross a three kilometer jungle road to reach the main road before getting any transport facility. Often we are dependent on male members to arrange a vehicle. Sometimes situations compel us for home delivery as it saves time and money despite the high risk (Savita, Nuakhejuria village).

Our local custom is such that our elders and husbands decide money matters. Decision on expenditure is not limited to pregnancy and delivery, but choice for taking care of the child dominates over expenditure on women's care. Hence, before delivery, we want to spend only a minimal amount (Dhuli, Phulta village).

Focus Group Discussions were carried out among the women and the community members. It was found that they are not convinced with the impact of antenatal care in reducing the risk of women attending fewer antenatal appointments, as even without ANC, a woman is able to deliver normally. 


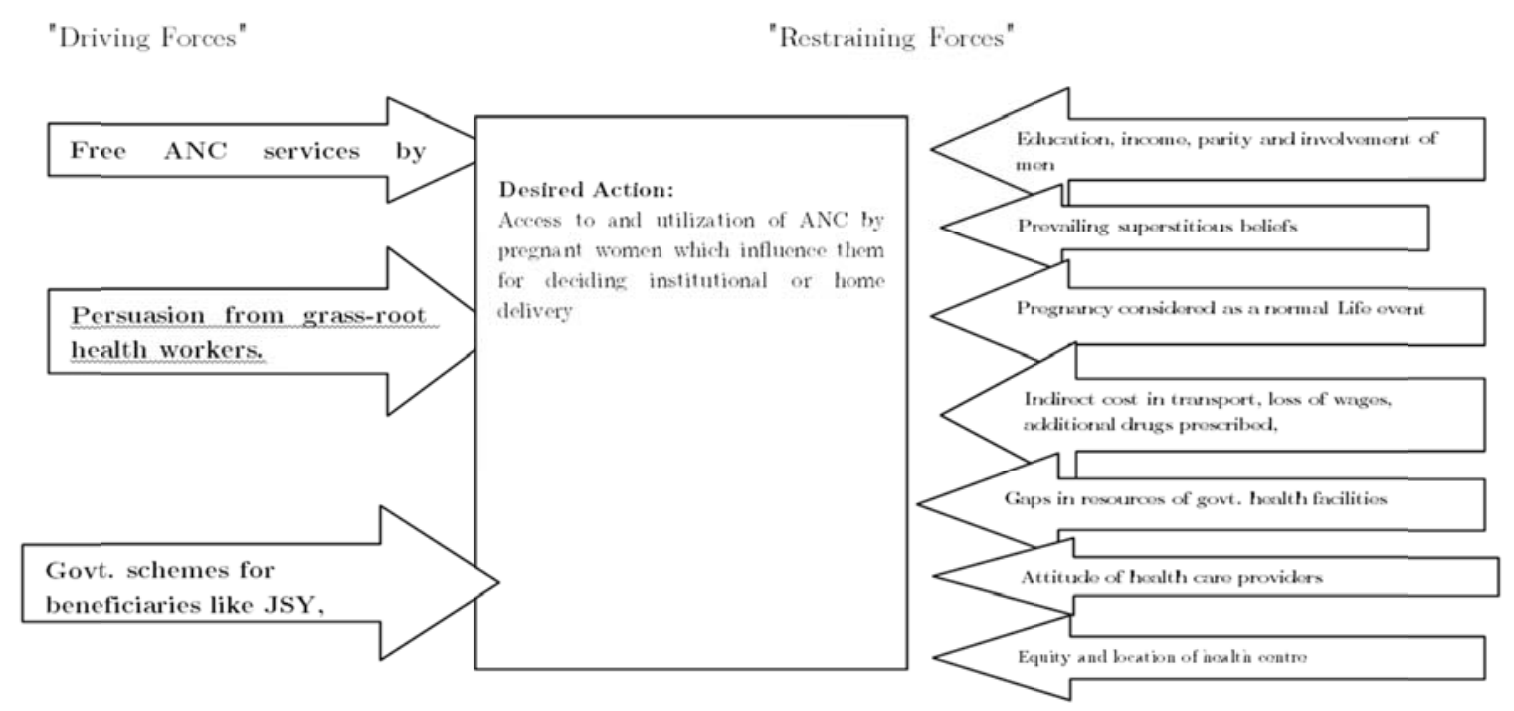

Figure. Force Field Analysis of Utilization of Antenatal Care Services by women

\subsection{Role of ANC in Deciding Place of Delivery}

Analysis was done from the quantitative data regarding the number of ANC visits made by the women helps in deciding place of delivery. The rate of $>3 \mathrm{ANC}$ visits was high (87.7\%) among women delivered at the institution compared to those delivered at home. This concluded that antenatal checkups influence the decision of women to deliver at institution. [Chi square $=21.252$, with 1 degree of freedom. The two-tailed $p$ value was less than 0.0001]. The association between antenatal checkups and place of delivery was considered to be extremely statistically significant. However, it was observed that $21.6 \%$ women had delivered at home and the rest had adopted for institutional delivery (table 2). The respondents reported that they faced transport problems and most of them did not know about the JSY scheme at all. Qualitative understanding also supplemented this observation.

Table 2 : Importance of ANC and Place of Delivery

\begin{tabular}{c|c|c|c}
\hline ANC & Home(70) & Institutional(250) & Frequency (\%) \\
\hline$<3 \mathrm{ANC}$ & $33.80 \%(48)$ & $66.20 \%(94)$ & $100 \%(142)$ \\
\hline$>3 \mathrm{ANC}$ & $12.30 \%(22)$ & $87.70 \%(156)$ & $100 \%(178)$ \\
\hline
\end{tabular}

\subsection{Discussion}

Although many health programmes have not been able to adapt contextual barriers to the use of health services, the important determinant emerging from the study was equity in accessing ANC due to locational advantage. The influence of grass root level workers and increased awareness among the women led to better antenatal care services utilization which helped them to decide institutional delivery. While in the social world of care and cure, the grass-root health workers and patients may have different health perspectives and therapeutic agendas [13], the medical setting in the hospital creates conditions under which every mode of communication and thinking initially takes precedence over formal concerns with production of objective medical knowledge [14]. What is further instilling in women the idea of going for institutional delivery is that the cutting-edge health professionals working in sub-centre as a last-resort health infrastructure, are constantly mediated with cultural impediments and social infrastructure. However, the very causes of their vulnerability also prevent them from receiving adequate care in pregnancy. Social barriers often blunt the cutting edge with perpetuating hindrance between the medical/scientific knowledge and the social construct. The restraining forces at 
different stages of decision-making limit accessibility and utilization of health care facility for institutional delivery. It was observed that income does not influence the number of ANC visits. While in other studies, the poor women were less likely to attend all antenatal visits than their respective nonpoor counterparts. In addition, it was found that the literacy status was directly proportional to number of antenatal visits made by the women. Similar findings were noted in other studies [15, 16]. In a national household survey in South Africa [17], it was found that the poorest women had the highest ANC coverage $(98.8 \%$ ) although only $39.6 \%$ of them attended these services before 20 weeks' gestation. Parity was inversely proportional to the rate of antenatal visits made by women; similar findings were observed in two other studies $[12,18]$. Out of pocket expenditure on health care was also identified as an important restraining force in utilizing the antenatal care services.

Divergent etiological concepts and perceived hospital culture and care were equally important determinants to control maternal healthcare-seeking behavior in Ganjam and Gajapati district, as has been shown for other countries $[19,20]$. The community members are not convinced with the impact of antenatal care in reducing the risk of women attending fewer antenatal appointments, as even without ANC, a woman is able to deliver normally. Similar observations made from Cochrane review of RCT shows that late, infrequence or nonattendance at antenatal services with adverse maternal outcomes is limited $[6,21]$. The study provided the evidence that perceived attitudes of women towards supernatural healing further reduce their readiness to seek care at the health-facility level. However, more ANC visits increase institutional deliveries among the study population.

\section{Conclusion}

Though the Indian government is doing all its efforts to encourage people to utilize the antenatal care services by providing these services free of charge and also giving monetary benefits to the users belonging to the economically weaker sections of the society, the infrastructure of government health facilities needs improvement. Access to health services has to be guaranteed for all people irrespective of the rural urban divide. It is often difficult for women of those districts where patriarchal traditional values still prevail to use health services unless the provided services are culturally acceptable in practice. The most important and challenging need of the hour is 'women empowerment' in the society to access their rights. In a cultural atmosphere, the socio-spatial setting of a clinical encounter becomes one of the determinants of medical care acceptance. As a result, the discrepancies between the provider's and the recipient's perspectives and realities create problems at the very core of diffusion, acceptability, and utilization. From a service point of view, it is extremely important to know and consider this barrier and work towards overcoming it. A reorientation of services towards the specific needs of rural women is within the scope of existing healthcare services and people usually try hard to overcome these barriers if they consider the effort worthwhile. Even though access to health services has to be guaranteed, gender sensitive government systems will further improve utilization of services.

Acknowledgment. Author acknowledges the contribution of the Director, National Institute of Health and Family Welfare (NIHFW), Prof. R.M. Tripathy and his team at the Berhampur Medical College, Berhampur, Ganjam.

\section{References}

1. Villar J, Bergsjo P: Scientific basis for the content of routine antenatal care. I. Philosophy, recent studies, and power to eliminate or alleviate adverse maternal outcomes.Acta Obstet Gynecol Scand 1997, 76:1-14.

2. Lumbiganon P: Appropriate technology: antenatal care. Int J Gynaecol Obstet 1998, 63(Suppl 1):S91-S95.

3. World Health Organization, Department of Reproductive Health and Research, Family and Community Health: Integrated management of pregnancy and childbirth. Pregnancy, childbirth, postpartum and newborn care: a guide for essential practice. Geneva, Switzerland; 2003.

4. I.S. Yar'zever, I.Y Said: Knowledge and Barriers In Utilization Of Maternal Health Care Services In Kano State, Northern Nigeria. European Journal of Biology and Medical Science Research 2013 91), 1:1-14. 
5. Kenneth Finlayson, Soo Downe : Why Do Women Not Use Antenatal Services in Low- and Middle-Income Countries? A Meta-Synthesis of Qualitative Studies 2013 DOI: 10.1371/journal.pmed.1001373.

6. International Institute of Population Sciences. DLHS and Facility Survey. Mumbai: IIPS, 2007.

7. Janani Suraksha Yojana (JSY) Govt. of India, Ministery of Health \& Family Welfare, Maternal Health Division. New Delhi: Govt. of India. October 2006.

8. Indira Gandhi Matritva Sahyog Yojana - A Conditional Maternity Benefit Scheme Implementation Guideline for State Governments / Ut Administrations Ministry of Women and Child Development: Government of India. April 2011.

9. Neelanjana Pandey: Perceived Barriers to Utilization of Maternal Health and Child Health Services: Qualitative Insights from Rural Uttar Pradesh, India (2010). International Institute for Population Sciences, India.

10.National Rural Health Mission. Meeting People's Health Needs in Rural Areas. Framework of Implementation 2005 -2012. New Delhi: GOI; 2005.

11.Griffiths P., Stephenson R.Understanding users' perspectives of barriers to maternal health care use in Maharashtra, India Journal of Biosocial Science 2001 Jul;33(3):339-59.

12.Elias Ali Yesuf and Ronit Calderon: Disparities in the use of antenatal care service in Ethiopia over a period of fifteen years. BMC Pregnancy and Childbirth 2013, 13:131.

13.Kumar A, Mahapatro M. The cutting edge in the blunt space: an anthropological construct of auxiliary nurse midwives' social world in the community. Healthcare in Low-resource Settings 2013; 1:e10.

14.Juncker T, Vanneste AM. Emergency obstetric care: experience from Matlab and Abhoyanagar, Bangladesh. In: European Conference on Tropical Medicine, Ham- burg. Oxford: Blackwell Science; 1995.

15.Rifkatu Nghargbu: Equity in maternal and child health care services in Nigeria. African Economic Research Consortium May 2013.

16. Yuan Shen et al: Equity in use of maternal health services in Western Rural China: a survey from Shaanxi province. BMC Health Services Research 2014, 14:155.

17.Njeri Wabiri et al: Equity in Maternal Health in South Africa: Analysis of Health Service Access and Health Status in a National Household Survey, PLOS 2013 (6).

18.Stella Babalola and Adesegun Fatusi: Determinants of use of maternal health services in Nigeria - looking beyond individual and household factors. BMC Pregnancy and Childbirth 2009; 9:43 doi:10.1186/1471-2393-9-43.

19. Chapman RR. Endangering safe motherhood in Mozambique: prenatal care as pregnancy risk. Soc Sci Med 2003; 57: 355-374. doi: 10.1016/s0277-9536(02)00363-5.

20.Sarita PT, Tuominen R. Use of healthcare services in two rural communities in Tanzania Community. Dent Oral Epidemiol 1993; 21: $133-5$.

21.Grossmann-Kendall F, Filippi V, De Koninck M, Kanhonou L. Giving birth in maternity hospitals in Benin: testimonies of women. Reprod Health Matters 2001; 9: 90-98. doi: 10.1016/s0968-8080(01)90095-3. 